\title{
Passive acoustic methods for fine-scale tracking of harbour porpoises in tidal rapids
}

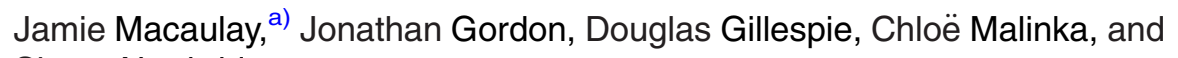 \\ Simon Northridge \\ Sea Mammal Research Unit, Scottish Oceans Institute, Institiud Chuantan na h-Alba, \\ University of St. Andrews, Gatty Marine Laboratory, East Sands, St. Andrews KY16 8LB, \\ Scotland, United Kingdom
}

(Received 9 August 2016; revised 9 December 2016; accepted 11 January 2017; published online 27 February 2017)

\begin{abstract}
The growing interest in generating electrical power from tidal currents using tidal turbine generators raises a number of environmental concerns, including the risk that marine mammals might be injured or killed through collision with rotating turbine blades. To understand this risk, information on how marine mammals use tidal rapid habitats and in particular, their underwater movements and dive behaviour is required. Porpoises, which are the most abundant small cetacean at most European tidal sites, are difficult animals to tag, and the limited size of tidal habitats means that any telemetered animal would be likely to spend only a small proportion of time within them. Here, an alternative approach is explored, whereby passive acoustic monitoring (PAM) is used to obtain fine scale geo-referenced tracks of harbour porpoises in tidal rapid areas. Large aperture hydrophone arrays are required to obtain accurate locations of animals from PAM data and automated algorithms are necessary to process the large quantities of acoustic data collected on such systems during a typical survey. Methods to automate localisation, including a method to match porpoise detections on different hydrophones and separate different vocalising animals, and an assessment of the localisation accuracy of the large aperture hydrophone array are presented. (C) 2017 Acoustical Society of America.
\end{abstract}

[http://dx.doi.org/10.1121/1.4976077]

[WWA]

Pages: $1120-1132$

\section{INTRODUCTION}

In many parts of the world, there is an increasing interest in generating renewable, low-carbon electricity in the marine environment. The prospect of harnessing power from tidal flows, which have the great advantage of being highly predictable, is being pursued in several regions with large tidal ranges and strong currents (Toke, 2011). Most proposed tidal generators have large, exposed, freely rotating blades and tip speeds may reach up to $12.5 \mathrm{~ms}^{-1}$. The potential risk for fish, birds, and marine mammals to collide with these blades, resulting in possible injury or death, is poorly understood and therefore considered by most regulators a primary conservation and welfare concern (Wilson et al., 2007). As the industry expands, the large scale deployment of tidal turbines and resulting inevitable increase in anthropogenic activity could also displace such animals (Frid et al., 2012). Little information exists on the interactions between tidal rapid areas and marine megafauna and thus the potential consequences of such habitat exclusion are not known (indeed a recent comprehensive review is titled "Confusion Reigns"; Benjamins et al., 2015). Of the multiple information gaps which exist in this area, the fine scale underwater movements and depth distributions of marine megafauna is perhaps least understood, yet forms a key parameter in assessing both collision risk (Thompson and Lonergan, 2015) and habitat usage.

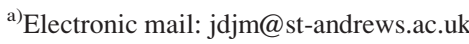

The most common marine mammal species in inshore waters of Europe and North America is the harbour porpoise, Phocoena phocoena (L.) (Hammond et al., 2013; Waring et al., 2015). In Europe, harbour porpoises are listed under Annex II and IV of the EU Habitats Directive (European Commission, 1992) which requires EU Member States to assess and address potential conservation threats, and in the US and Canada they are protected by the "Marine Mammal Protection Act and the Species At Risk Act" (MMPA, 1972). Harbour porpoises are small, undemonstrative and shy animals making many visual research methods difficult to apply, especially in tidal rapids which, due to fast moving currents, often have significantly higher sea states than surrounding areas. However, harbour porpoises are also highly vocal animals, producing characteristic narrow bandwidth $(16 \mathrm{kHz}-3 \mathrm{~dB}$ frequency width), high frequency (centred at $130 \mathrm{kHz}$ ), highly directional (beam pattern $9.5^{\circ}$ to $16^{\circ}$ at $-3 \mathrm{~dB}$ ) clicks (Mohl and Andersen, 1973; Verfuß et al., 2009; Au et al., 1999; Koblitz et al., 2012; Kyhn et al., 2013). The theoretical maximum on-axis detection range for these vocalisations is restricted to $\$ 500 \mathrm{~m}$ by relatively modest maximum source level (Villadsgaard et al., 2007) and a high absorption coefficient at $130 \mathrm{kHz}$ (Ainslie and Mccolm, 1998) (assuming spherical spreading and a detection threshold of $120 \mathrm{~dB}$ re $1 \mu \mathrm{Pa}$ ). On the other hand, porpoise clicks are highly characteristic and produced in frequency bands where ambient noise levels are generally low. These narrow band high frequency (NBHF) clicks provide an additional method for detecting porpoises 
underwater which is largely unaffected by sea state, time of day and weather conditions. This paper describes the development of passive acoustic monitoring (PAM) methods to localise and track harbour porpoise underwater in tidal rapid areas in order to provide detailed information on their depth distribution and underwater movements.

Recording accurate geo-referenced positions of marine mammals underwater is not a trivial problem. Animal-borne tags with depth and orientation sensors and/or global positioning systems (GPS) are an initial obvious choice (e.g., Linnenschmidt et al., 2013; Wisniewska et al., 2016). However, when such methods are applied to a geographically restricted and atypical habitat, the likelihood that any tagged animal will spend a large proportion of their time in the study area is low. Thus, a tagging programme to collect a significant volume of data on diving behaviour of porpoises in tidal rapid areas would be likely to be prohibitively expensive. PAM has the potential to provide an alternative approach to obtain fine scale information on behaviour, targeting animals within a specific geographic area of interest, such as tidal rapids.

Widely spaced (or "large aperture") hydrophone arrays have been used for decades to track the movements of cetaceans underwater (e.g., Watkins and Schevill, 1972; Møhl et al., 2000; Miller and Dawson, 2009; Wiggins et al., 2012). By analysing the time of arrival differences (TOADs) between a vocalisation detected on several dispersed hydrophones, it is possible to determine the position of the vocalising animal. Such systems have been used to determine locations of NBHF species (e.g., Ural et al., 2006; Villadsgaard et al., 2007; Kyhn et al., 2013). However, these studies all used rigid hydrophone arrays with dimensions of a few meters and therefore the range at which accurate localisations were possible was restricted to tens of meters. Although these arrays were appropriate for their respective studies, for this application, a PAM system capable of providing accurate animal locations at ranges up to a few hundred meters was required and thus a significantly larger hydrophone array which could be deployed in strong currents was required.

Large aperture vertical linear hydrophone arrays can be deployed from a drifting vessel and have been used in the past to localise the depth of bottlenose dolphins in tidal areas (Hastie et al., 2006). Crucially, such systems can be on the order of tens or hundreds of meters long (e.g., Holt et al., 2009; Heerfordt et al., 2007) providing accurate localisations at larger ranges. Any linear array can only provide locations in two dimensions (see $\mathrm{Au}$ and Hastings, 2008) and in the case of a vertical linear array, these would be the range and depth of a vocalising cetacean. However, information such as the orientation of animals in a tidal stream and fine-scale movements in relation to bottom topography is important in understanding how tidal habits are utilised and is lost if animals are only located in two dimensions. In addition, significant errors in localisation can be introduced if a linear vertical array moves off the vertical axis - a circumstance which is likely to occur when the supporting vessel or buoy drifts with the wind and/or if currents vary with depths.

This paper explores the use of a large aperture vertical hydrophone array drifting in tidal rapids to obtain fine scale three-dimensional (3D) geo-referenced porpoise tracks. ${ }^{1}$ The accuracy of the system is extensively tested by broadcasting simulated porpoise clicks from known locations and depths. Automated methods to analyse the large quantities of data collected the array during a typical survey are presented.

\section{MATERIALS AND METHODS}

\section{A. Using large aperture hydrophone arrays in tidal rapids}

There are several practical difficulties which must be considered when designing a large aperture array to localise harbour porpoises.

Strong and differential currents in tidal rapids mean that large and heavy structures would be required to deploy an array rigidly on the seabed. Anchoring a buoy or vessel in strong currents would be difficult and the array configuration would be hugely distorted by the current. A drifting array is therefore a much more cost effective and practical option. However, any drifting array in tidal rapids must be capable of being quickly deployed and recovered as fast currents are likely to carry it into dangerous and/or shallow-water areas. Free hanging weighted vertical cable arrays are a practical option as they can be quickly recovered either by hand or using a winch.

Wave action, strong differential currents and wind moving the research vessel against tide can cause a free hanging array, even if substantially weighted, to move through the water. This can cause significant deformation of the array creating uncertainty in the position of hydrophone elements. To maintain localisation accuracy the locations of all the hydrophones in the array must be measured with fine temporal resolution, i.e., multiple measurements per second.

A simple linear vertical system which moves off the vertical axis will lose resolution in depth, even if the angle of the array and therefore the position of hydrophones are known accurately. This is because a localisation from any linear hydrophone array restricts the position of a source to a circle of possible locations. The circle is centred on and perpendicular to the linear array. In the case of a vertical linear array, the radius of the circle represents the horizontal range and the depth directly corresponds to the depth of a source. If a vertical array moves off the vertical angle, even if the position of all hydrophone is known, a fundamental uncertainty is introduced in depth and range, i.e., the source is still located on a circle but that circle is now at an angle. Thus, any linear array must remain close to vertical to provide accurate depth information. Therefore, for any deployment in tidal rapids an array must be designed in such a way that the depth information can still be calculated if the array moves off the vertical axis, which is inevitable in an environment with high flow. This is achieved by ensuring at least some hydrophone elements are not in a straight line, i.e., breaking the linearity of the array.

Perhaps the most significant design consideration is determining the optimal spacing between hydrophone elements in the array. Generally, an array with larger spacings between hydrophones will provide more accurate locations as the errors due to uncertainty in hydrophone positions, and 
time of arrival measurements, are proportionally smaller. However, echolocating cetaceans, including harbour porpoises, produce highly directional clicks (Au et al., 1999; Koblitz et al., 2012) and click source amplitudes are under behavioural control and can vary substantially (Deruiter et al., 2009). Porpoise clicks might thus be thought of as a narrow beam flashlight constantly varying in intensity and width, rather than a uniform spherical pulse (Wisniewska et al., 2015). This narrow beamwidth presents several challenges when large aperture hydrophone arrays are used because it is likely that only a subset of hydrophones will be ensonified by an animal's directional sonar beam at any one time. For a 2D location (depth and range) to be determined, a minimum of three hydrophone elements must detect a vocalisation and for a 3D location at least four elements, distributed in three dimensions, are required (Wahlberg et al., 2001). The spacing of hydrophones in arrays is therefore a trade-off. Hydrophone elements must be spaced sufficiently close together for a minimum number to be consistently ensonified by directional vocalisations, but sufficiently separated to allow accurate localisation at useful ranges.

\section{B. Vertical array design}

Several designs of hydrophone array were tested; however only the final iteration is discussed here. This consisted of a 30-45 $\mathrm{m}$ vertical array with 6-8 hydrophones spaced between 4 and $11 \mathrm{~m}$ apart and a tetrahedral cluster of four hydrophones with elements separated by $50 \mathrm{~cm}$ (the "quad array") mounted rigidly on the vessel with an offset of $\sim 7.5-8 \mathrm{~m}$ from the vertical array (Fig. 2). The quad array provided an unambiguous vector which, when combined with the range and depth from the vertical array, allowed 3D coordinates to be determined. To minimise flow noise and provide mechanical protection the hydrophone elements were housed individually inside small oil-filled polyurethane tubes, each of which was attached to a non-stretch Kevlar rope that was kept taut by a terminal weight $(80 \mathrm{~kg})$.

Both the quad array and vertical array could be quickly deployed and recovered ( $<3 \mathrm{~min})$. The vertical array sections were hung from the side of a vessel and the windlass on the anchor winch was used to recover the weight whilst the array itself was brought on board by hand. The quad array was rigidly attached to the boat's hull with a quick release mechanism that allowed for rapid recovery. OpenTag ${ }^{\mathrm{TM}}$ inertial measurement units (IMUs) from Loggerhead Instruments (Loggerhead Instruments, Sarasota, FL), equipped with a 3D accelerometer, 3D magnetometer, and 3D gyroscope, as well as sensors for pressure and temperature, were attached at regular intervals along the vertical array. These measured heading and pitch at each location, allowing the shape of the vertical array to be reconstructed, and so the position of hydrophones to be determined. A Hemisphere VS101 vector GPS (Hemisphere, Scottsdale, AZ) was used to record the heading and position of the vessel and an IS-2-30 inclinometer (Level Developments, Chicago, IL) was used to measure the vessel's pitch and roll. The combined data from these sensors allowed a time series of the 3D location of all hydrophones to be calculated every $0.5 \mathrm{~s}$.
Both arrays used Magrec HPO3 hydrophones (Magrec, Devon, UK). Each of these consisted of a spherical $9 \mathrm{~mm}$ diameter ceramic with a sensitivity of -218.69 to $-194.3 \mathrm{~dB}$ re $1 \mathrm{~V} / \mu \mathrm{Pa} @ 150 \mathrm{kHz}$, connected to a Magrec HPO2 pre-amplifier (with gains of either 28 or $40 \mathrm{~dB}$ and $20 \mathrm{kHz}$ high pass filter). Signals from hydrophones were further amplified and filtered on the vessel using a custom four-channel ETEC (ETEC, Frederiksvaerk, Denmark) and two stereo Magrec HP27 amplifiers; high-pass filters of $20 \mathrm{kHz}$ were typically applied to reduce low and medium frequency noise. National Instruments (NI) data acquisition (DAQ) cards $(6251,6351$, and 6356) were used to digitise the signals at sample rates ranging between 500 and $1000 \mathrm{kHz}$ (National Instruments, Austin, TX). NI cards were used in a master-slave configuration, whereby all acquisition was from a single clock pulse (the master) guarantying synchronisation over-all channels. In 2014, both the amplifiers and NI DAQ cards were replaced with three synchronised four-channel SAIL DAQ cards ( $\mathrm{St}$ Andrews Instrumentation Limited, www.sa-instrumentation.com). These have inbuilt software adjustable amplifiers, filters and DAQ abilities. All recordings were saved as WAV files using PAMGuard (Gillespie et al., 2009) (www.pamguard.org).

\section{Localisation accuracy trials}

The location accuracy that could be achieved with the acoustic array was tested by broadcasting simulated porpoise clicks at known locations and depths.

A Matlab (The Mathworks Inc., Natick, MA) script was written to produce a single channel WAV file containing bursts of 25 simulated porpoise clicks (length: $0.1 \mathrm{~ms}$, frequency: $140 \mathrm{kHz}$, envelope: Gaussian). This was output through an NI 6252 DAQ card at $1 \mathrm{~V}_{\mathrm{p}-\mathrm{p}}$ using PAMGuard. The signal was amplified by a Sony XPLOD $1200 \times$ stereo amplifier (Sony, Tokyo, Japan) and then projected from a transmit transducer, Neptune Sonar HS150 hydrophone (Neptune Sonar Ltd., East Yorkshire, UK), on a $30 \mathrm{~m}$ cable. The broadcast system was operated from an inflatable boat which could then drift at different ranges from the array while the deployment depth of the transducer was adjusted. An Aladin dive computer (ScubaPro, El Cajon, CA) and OpenTag $^{\mathrm{TM}}$ were used to record the depth of the transmit hydrophone and a GlobalSat BU-353-S4 GPS (GlobalSat, Davie, FL) logged by PAMGuard provided a record of the position of the boat carrying the sound source.

\section{Hydrophone calibration}

All hydrophones on the array were calibrated by comparing received RMS voltages from a broadcast source to a calibrated Reson TC4013 hydrophone and Reson VP2000 amplifier (Teledyne, Slangerup, Denmark). The calibrated hydrophone was mounted next to each hydrophone and series of tones, from 20 to $200 \mathrm{kHz}$, were output at a range of $20 \mathrm{~m}$ using the broadcast system described above. 


\section{ANALYSIS}

\section{A. Localisation algorithms}

The theory underpinning the process of localising a vocalising animal using an array of receivers is relatively straight forward. A sound (porpoise click) is detected on multiple receivers (hydrophones), the time delays between the click arriving at different hydrophone are measured, and from this, a location can be determined, either by direct calculation or using an iterative search algorithm.

\section{Hyperbolic localisation}

The most common way to calculate the position of a sound source from a set of time delays is via hyperbolic localisation, i.e., to directly calculate from observed time delays via a set equation (Watkins and Schevill, 1972). This method has the great advantage of being computationally efficient. However, it cannot automatically deal with ambiguous results, propagating errors can be complex (Wahlberg et al., 2001) and different equations have to be constructed for linear, planar, and volumetric arrays ( $\mathrm{Au}$ and Hastings, 2008).

\section{Iterative search algorithms}

With the advance of modern computing, it is now practical to use localisation algorithms based on what is commonly termed as "the forward problem." Instead of trying to directly calculate parameters (the source location) from given observables (time delays), the problem is approached from the other direction; by answering the question, what time delays would be produced from a source in a given location?

Assuming a refraction free environment, it is straightforward to calculate the time it would take for a sound wave produced by a source at $s=\left(s_{x}, s_{y}, s_{z}\right)$, travelling at a speed of $c$ to reach a hydrophone $i$ located at $r=\left(r_{x}, r_{y}, r_{z}\right)$ using

$$
T(i)=\sqrt{\frac{\left(r_{x}(i)-s_{x}(i)\right)^{2}+\left(r_{y}(i)-s_{y}(i)\right)^{2}+\left(r_{z}(i)-s_{z}(i)\right)^{2}}{c^{2}}} .
$$

From this, the expected time delay between two hydrophones can be found by calculating the time from the source to each hydrophone and subtracting one from the other. Thus, it is possible to calculate all the time delays expected between all elements on an array for a source at a given location. A $\chi^{2}$ value can determine the extent to which these time delays match time delays from a real set of observed data by the function:

$$
\chi^{2}=\sum \frac{\left(\tau_{\mathrm{obs}}(i j)-\tau_{\mathrm{calc}}(i j)\right)^{2}}{\varepsilon^{2}},
$$

were $\tau_{\mathrm{obs}}(i j)$ is the actual observed time delay between hydrophones $i$ and $j, \tau_{\text {calc }}(i j)$ is the calculated time delay between hydrophones $i$ and $j$ from an acoustic source at some point in space and $\varepsilon$ represents the expected error in observed data. For example, for a four hydrophone array, $i=1,1,1,2,2,3$ and $j=2,3,4,3,4,4$.

Various algorithms exist to sample large spatial volumes to find a location (or locations) which minimise the $\chi^{2}$ value, i.e., find the most likely location of the acoustic source. Such algorithms are generally more computationally intensive than directly solving via set equations but can also provide more reliable information on potential errors and ambiguities. Two such algorithms are discussed below.

a. Simplex. The downhill simplex optimisation method (Nelder and Mead, 1965) is a common optimisation function which can be used to minimise the $\chi^{2}$ value in any number of dimensions. In three dimensional space, the simplex can be visualised as a tetrahedron which, at each stage in the search process, can stretch, contract and reflect through its own centre until it surrounds the most likely solution and contracts to a point. Once the size of that point reaches a predetermined minimum size, the algorithm stops. Although it is fast, a disadvantage of the simplex algorithm is that it returns no error estimate. A good description of the simplex algorithm can be found in Press et al. (1988).

b. Markov chain Monte Carlo. Markov chain Monte Carlo (MCMC) is a simulation technique which can be used to solve a wide variety of problems. For localisation, a random walk MCMC algorithm, Metropolis-Hastings algorithm (Metropolis et al., 1953), was implemented. This utilises a series of random jumps in space to arrive at the most likely source location. From an initial random point, a jump is made to a new point via a random jump function; in this case, a random Gaussian number generator determines new $x, y$, and $z$ coordinates to jump to. After each new jump, $\chi^{2}$ is calculated for the time delays that would be generated by an animal at the new jump location. If $\chi^{2}$ is lower than the previous point in space, the jump is executed. If it is not, then the jump is only executed with a probability of

$$
p=e^{-\left(\Delta \chi^{2} / 2\right)}
$$

where $\Delta \chi^{2}$ represents the difference in $\chi^{2}$ values between the previous and new jump point. If a jump is unsuccessful, a new random jump is calculated and the process repeats. As the number of iterations grows, a chain of jumps is created, which converges to a volume in space where parameter values result in a low $\chi^{2}$ value, i.e., where observed and calculated data are similar. Thus, in an acoustic localisation, a chain will converge in space to the most likely position of the acoustic source and create a "cloud" around the likely source location. For a linear vertical array, this should be a doughnut-shaped cloud of points with a well-defined depth and range. For a 3D array, the cloud will be centred on a specific point. The mean position of points within the cloud represents the location, and the standard deviation of points directly corresponds to the standard error in location (Chib and Greenberg, 1995).

A typical MCMC localisation algorithm will run many chains, each starting at a different random location. The convergence of multiple chains to the same location is a good 
indication of a valid result, however random start locations also allow for different chains to converge to different possible localisation positions if ambiguities exist. If such ambiguities do exist, then the average location of points will simply give the average positions of different ambiguous results. Therefore, a clustering algorithm is required. A $k$ means square (MacQueen, 1967) clustering algorithm was used which assumed no more than five possible clusters and set a minimum value of $5 \mathrm{~m}$ absolute distance between different clusters before they were considered a single result.

MCMC methods provide a useful visualisation of localisation results, accurately propagate errors and create clusters of results if ambiguities in location exist. It therefore provides a flexible and informative, albeit computationally demanding, method to localise sound sources.

\section{B. Practical issues in localising harbour porpoises in tidal rapids}

There are a number of general environmental and physical factors which must be considered when using PAM to detect, classify and localise marine mammals and these have been discussed in many publications (e.g., Au and Hastings, 2008; Zimmer, 2011). Localizing harbour porpoises with large aperture hydrophone arrays in energetic tidal habitats presents some additional practical and analytical challenges.

\section{Click match uncertainty}

Any TOAD based localisation method requires the same signal to be identified on all or a subset of elements within an array. When the distance between elements is small and potential signals arrive relatively infrequently, it is comparatively straight forward to identify the same signal on all the hydrophones in an array. However, there is the potential for match uncertainty when the time between potential signals becomes similar to or less than the time of flight between elements in an array. Match uncertainty occurs when a detection of a transient on one hydrophone channel may be wrongly matched with a detection of a different transient on another hydrophone channel. This problem increases as arrays get larger and the time of flight between elements increases. A number of factors which are particularly relevant to harbour porpoises in tidal rapids habitats contribute to the problem of match uncertainty. These are as follows:

(1) High vocalization rates. Many tidal rapid areas are thought to be important areas for foraging (Pierpoint, 2008; Benjamins et al., 2015). During foraging harbour porpoises click rates increase and in the final phase of prey capture reach $\sim 600$ clicks per second [an inter click interval (ICI) of $1.67 \mathrm{~ms}$ ] (Verfuß et al., 2009)

(2) Two or more individuals vocalising at the same time. Harbour porpoise show a habitat preference and/or are aggregate in unusually high numbers in at least some high energy tidal sites (Goodwin, 2008; Marubini et al., 2009; Gordon et al., 2011) and therefore simultaneously detecting multiple individuals is likely.
(3) Reverberation. Tidal habitats are often shallow and therefore reverberant environments resulting in strong echoes detected from the sea surface and sea bed.

An initial intuitive approach might be to match detections on different hydrophone based on the waveform, amplitude, and/or spectral characteristics of detected clicks. In the case of harbour porpoises, due to their narrow beam profiles, the same click detected at different angles relative to the porpoise can have very different waveforms and amplitudes and clicks from different individuals very rarely show consistent differences. Thus, an approach based on spectral or temporal characteristics will do little to match clicks on different hydrophones.

Instead a different approach is required. It is obvious that detections on different hydrophones are only potential matches if they occur within a time window determined by the distance between the hydrophones, $d$, and the speed of sound, $c$, i.e., a detection is only a possible match if it is $d / c$ seconds before or after a primary detection. If there are multiple detections within a time window on different hydrophones, then there are many possible combinations of transient detections any of which could be the direct arrival of the primary detected signal. An example of this is shown in Fig. 1.

To solve match uncertainty, the correct combination of time delays needs to be determined. One approach is simply to calculate the sound source location for every possible combination of detections. The combination of TOADs which produce a source location with the lowest $\chi^{2}$ value (best fit to the localisation model) is selected as the most

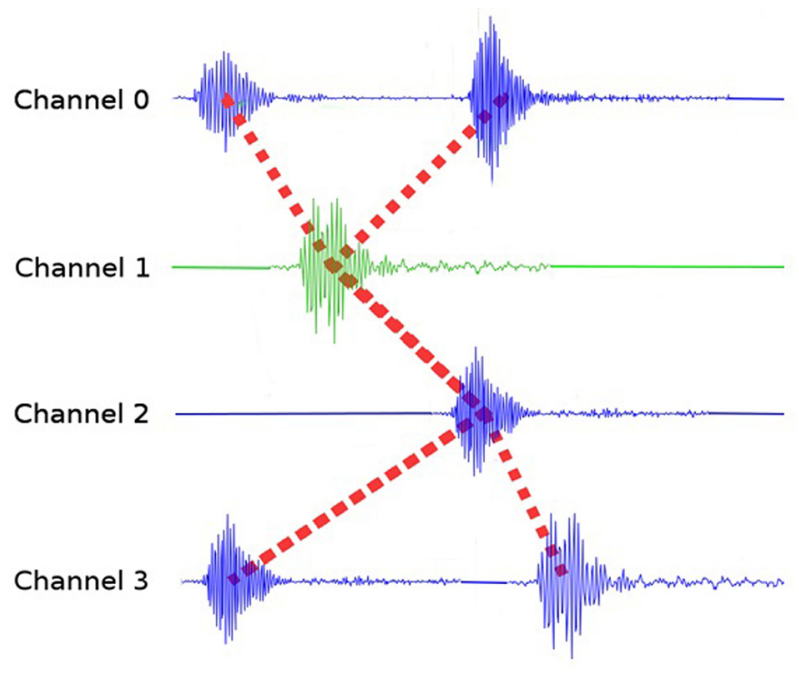

FIG. 1. (Color online) Example of match uncertainty. A detected click on channel 1 should also be detected on channels 0,2 , and 3 . As the position of the animal is unknown, to find the same click on another channel it is necessary to look $t$ seconds before and after the primary click were $t$ is a time related to the distance between hydrophones. In this time window there may be several clicks detected due to a variety of factors including echoes, high click rates or other vocalising animals. As porpoise clicks from different individuals and echoes are essentially indistinguishable, finding the correct combination of clicks is difficult. One solution is to localise every possible combination, shown here by dashed lines. Incorrect combinations will either be localised to unrealsitic locations, e.g., above the sea surface, or poorly fit the localisation algorithm used, resulting in a high $\chi^{2}$ value. 
likely location. Many of the calculated positions resulting from incorrect combinations and/or localisation of echoes will be unrealistic, located above the sea surface or at an improbable range and/or depth; these are discarded.

This method requires a position to be calculated for every combination of detections. The number of combinations for a ten channel system can quickly reach thousands of locations, and therefore computationally efficient methods of localisation are required. Hyperbolic and simplex algorithms are fast enough to allow for many thousands of combinations to be calculated in a few seconds. However, using these methods alone precludes some of the advantages from the more processor intensive iterative approaches, such as MCMC. Therefore, a hybrid algorithm was developed: faster algorithms were used to calculate the most likely combination of detections and then those combinations were localised with an MCMC based algorithm. This approach was named "mimplex."

The mimplex algorithm works as follows:

(1) For a given porpoise click, find all possible matching clicks on different hydrophones.

(2) Calculate all possible time delay combinations.

(3) Localise every time delay combination using hyperbolic and simplex algorithms.

(4) Using Eq. (2) calculate the $\chi^{2}$ value for each localisation. The combination with the lowest $\chi^{2}$ is deemed the correct combination and localised with MCMC.

(5) The result is saved and the algorithm moves to the next click. Select the next click in the series and go to step 1.

(6) After processing has finished, discard results if above sea level or at an unrealistic range or depth or if $\chi^{2}$ is higher than a predefined threshold.

For each localisation, the mimplex algorithm requires a primary channel. In this case, there are two approaches. The first is to use predefined channel on the array as the primary channel. If a click is not detected on that channel then it is not localised and therefore some useful localisations are potentially missed. The second approach is to use a dynamic primary channel. However, this requires that matched clicks are removed from further localisation attempts. This involves a decision on whether a localisation is valid during, rather than after, processing (e.g., it is not advisable to remove matched clicks from further localisation if the current localisation is $500 \mathrm{~m}$ above sea level and therefore invalid) and so introduces a significant extra level of complexity.

Here, for simplicity, a hydrophone midway on the vertical array was used as the predefined primary channel under the assumption that most clicks which ensonified a minimum number of hydrophones on the vertical array for a successful localisation would most likely be detected on a mid-array hydrophone. This assumption would not hold for a significantly longer array with more hydrophones and thus developing a robust dynamic primary channel system is a focus of further work.

\section{Tracking dives}

Having calculated the locations for individual clicks the next step is to join these into a series of tracks showing the movement and behaviour of individual animals. The high frequency and therefore rapid attenuation of NBHF clicks combined with highly directional vocalisations means that the probability of a harbour porpoise ensonifying a sufficient number of hydrophones on a large aperture array for a successful localisation (three for a 2D location and four for a 3D location), is dependent on an individual animal's range, depth and orientation. This and the fact that porpoises may not vocalise continuously mean that a typical acoustic encounter with a porpoise will at best provide a scatter of localisation points representing only fragments of a complete dive. In addition, these localisation points are not evenly distributed in time; changes in ICI will result in more or fewer localisations per second. A tracking algorithm is therefore required to perform the following tasks.

(1) Separate the clicks and tracks of different animals vocalising at the same time.

(2) Interpolate the tracks of individual animals.

(3) Smooth interpolated tracks to compensate for localisation errors and gain more accurate insights into animal swim speed and orientation.

This is relatively simple for a single animal, merely requiring a "joining of dots" and then smoothing and interpolation of the resulting track. However, the problem is more complex when localisations of clicks of several animals are calculated at the same time. As discussed above, clicks of different individuals cannot be distinguished by their acoustic characteristics and therefore tracks must be identified based on location rather than acoustic information. This type of pattern recognition problem, often referred to as multi target tracking, is common and several solutions exist (e.g., Berclaz et al., 2011); the approach adopted here was to use a state estimation technique, a 3D Kalman filter (Kalman, 1960) combined with a matching algorithm, Hungarian method (Kuhn, 1955), to track multiple animals simultaneously (Yussiff et al., 2014; Luetteke et al., 2012). In this model, the movement of a porpoise is described by

$$
\begin{aligned}
{\left[\begin{array}{c}
x_{k} \\
y_{k} \\
z_{k} \\
\dot{x}_{k} \\
\dot{y}_{k} \\
\dot{z}_{k}
\end{array}\right]=} & {\left[\begin{array}{llllll}
1 & 0 & 0 & t & 0 & 0 \\
0 & 1 & 0 & 0 & t & 0 \\
0 & 0 & 1 & 0 & 0 & t \\
0 & 0 & 0 & 1 & 0 & 0 \\
0 & 0 & 0 & 0 & 1 & 0 \\
0 & 0 & 0 & 0 & 0 & 1
\end{array}\right]\left[\begin{array}{c}
x_{k-1} \\
y_{k-1} \\
z_{k-1} \\
\dot{x}_{k-1} \\
\dot{y}_{k-1} \\
\dot{z}_{k-1}
\end{array}\right] } \\
& +\left[\begin{array}{c}
t^{2} / 2 \\
t^{2} / 2 \\
t^{2} / 2 \\
t \\
t \\
t
\end{array}\right] a+E x,
\end{aligned}
$$

where $x, y, z$ are the independent Cartesian coordinates of the porpoise and $\dot{x}, \dot{y}, \dot{z}$ are the velocity, or the derivative of $x, y, z$ with respect to time. $t$ is time, $a$ is a normally distributed acceleration of a typical harbour porpoise with a mean 
of 0 and a standard deviation of $\sigma_{a}$ and $k$ represents concurrent time steps ( $k=0,1,2,3, \ldots$ total steps). $E x$ is the process error/noise is defined by the covariance matrix,

$$
E x=\sigma_{a}{ }^{2}\left[\begin{array}{cccccc}
t^{4} / 2 & 0 & 0 & t^{3} / 2 & 0 & 0 \\
0 & t^{4} / 2 & 0 & 0 & t^{3} / 2 & 0 \\
0 & 0 & t^{4} / 2 & 0 & 0 & t^{3} / 2 \\
t^{3} / 2 & 0 & 0 & t^{2} & 0 & 0 \\
0 & t^{3} / 2 & 0 & 0 & t^{2} & 0 \\
0 & 0 & t^{3} / 2 & 0 & 0 & t^{2}
\end{array}\right] .
$$

Equation 4 can be rewritten as

$$
\bar{x}_{k}=F \bar{x}_{k-1}+G a_{k}+E x,
$$

where $F$ is defined as the state transition matrix and $G$ is the control input matrix and $\bar{x}_{k}$ is the position, velocity vector.

As position is the only measurement which can be made from localisation results, the measurement update model is

$$
z_{k}=\left[\begin{array}{cccccc}
1 & 0 & 0 & 0 & 0 & 0 \\
0 & 1 & 0 & 0 & 0 & 0 \\
0 & 0 & 1 & 0 & 0 & 0
\end{array}\right]\left[\begin{array}{c}
x_{k} \\
y_{k} \\
z_{k} \\
\dot{x}_{k} \\
\dot{y}_{k} \\
\dot{z}_{k}
\end{array}\right]+E z
$$

where $E z$ is the measurement error/observation noise defined by

$$
E z=\left[\begin{array}{ccc}
\sigma_{x}^{2} & 0 & 0 \\
0 & \sigma_{y}^{2} & 0 \\
0 & 0 & \sigma_{z}^{2}
\end{array}\right],
$$

where $\sigma_{x}, \sigma_{y}, \sigma_{z}$ are the standard deviations in position measurements $(x, y, z)$; these are calculated automatically by the mimplex algorithm for each localisation point.

Equation (7) can be rewritten as

$$
z_{k}=H \bar{x}_{k}+E z \text {, }
$$

where $H$ is defined as the observation matrix.

$F, G, H, E x$, and $E z$ form the basis of the Kalman filter which can be constructed as shown in Kalman (1960). On its own, the Kalman filter simply smooths a single animal track and will not perform well if multiple tracks are present. However the predictive component of a Kalman filter allows multiple instances to be used in conjunction with a Hungarian matching algorithm (Kuhn, 1955) to track multiple animals simultaneously. The algorithm works as follows:

(1) Data are binned into $0.5 \mathrm{~s}$ intervals. All positions are clustered using a MATLAB "cluster" function.

(2) A Kalman filter is started from each cluster in the first bin.

(3) Move to the next time bin.
(4) For all current tracks the next predicated state is calculated by $\bar{x}_{k}=F \bar{x}_{k-1}+G a_{k}$.

(5) All possible distances between observed animal positions and predicated states are calculated and input into a cost matrix. Localised positions are then assigned to predictions via a Hungarian matching algorithm.

(6) If a track has not been assigned to a new localisation, it is tagged with a "strike." If the number of strikes reaches a pre-defined number, it is stopped in step 10 .

(7) If a track has been assigned to a localisation which is greater than a defined maximum distance (in this case $15 \mathrm{~m}+10 \%$ of the localisation depth) from the end of the track, that detection is ignored and the track is given a strike.

(8) If a localised detection has not been assigned a track, or has been ignored because it is greater than the maximum allowed distance, a new track is started from that detection.

(9) For any track successfully assigned a detection, all strikes are removed.

(10) Any track which has more than the allowed number of strikes is stopped. The end of that track which is formed only by predictions is removed and the remaining section added to a list of completed track fragments.

(11) The Kalman filter for each current track is updated.

(12) Go back to step 3 and continue to last time bin.

Using this algorithm, it is possible to automatically generate track fragments from large quantities of data, a sample of which is shown in Fig. 6. The standard deviation in acceleration $\left[\sigma_{a}\right.$ in Eq. (5)] was $0.12 \mathrm{~ms}^{-2}$, a liberal estimate based on Teilmann et al. (2006) and the maximum number of strikes allowed for each track was 15 .

\section{Array movement error}

In any localisation calculation, error in receiver positions will propagate to an error in the localised positions of animals. Receiver movement is a major concern in flexible vertical array systems used in tidal currents. Arrays can deform substantially as a result of differential currents at different depths and the effects of wind on the surface supporting vessel. Therefore, simply assuming that an array remains vertical could introduce large errors in the localised positions under some conditions.

The five OpenTag ${ }^{\mathrm{TM}}$ orientation sensors on the array were attached with roughly regular spacing (Fig. 2). The orientation of the vertical array was therefore known at each orientation sensor. These data were used to reconstruct the most likely "shape" of the vertical array underwater and thus determine the most likely position of each hydrophone. To model the array shape the vertical array was spilt into $n$ (in this case $n=100)$ sections. Section 0 starting where the array connects to the vessel at $\left(x_{0}, y_{0}, z_{0}\right)=(0,0,0)$ and section $n$ ending at the deepest hydrophone. Each orientation sensor was located on a section between 0 and $n$. The unit vector of that section was provided by the angle recorded by the orientation tags. For the other sections without a sensor, the unit vector was defined as 


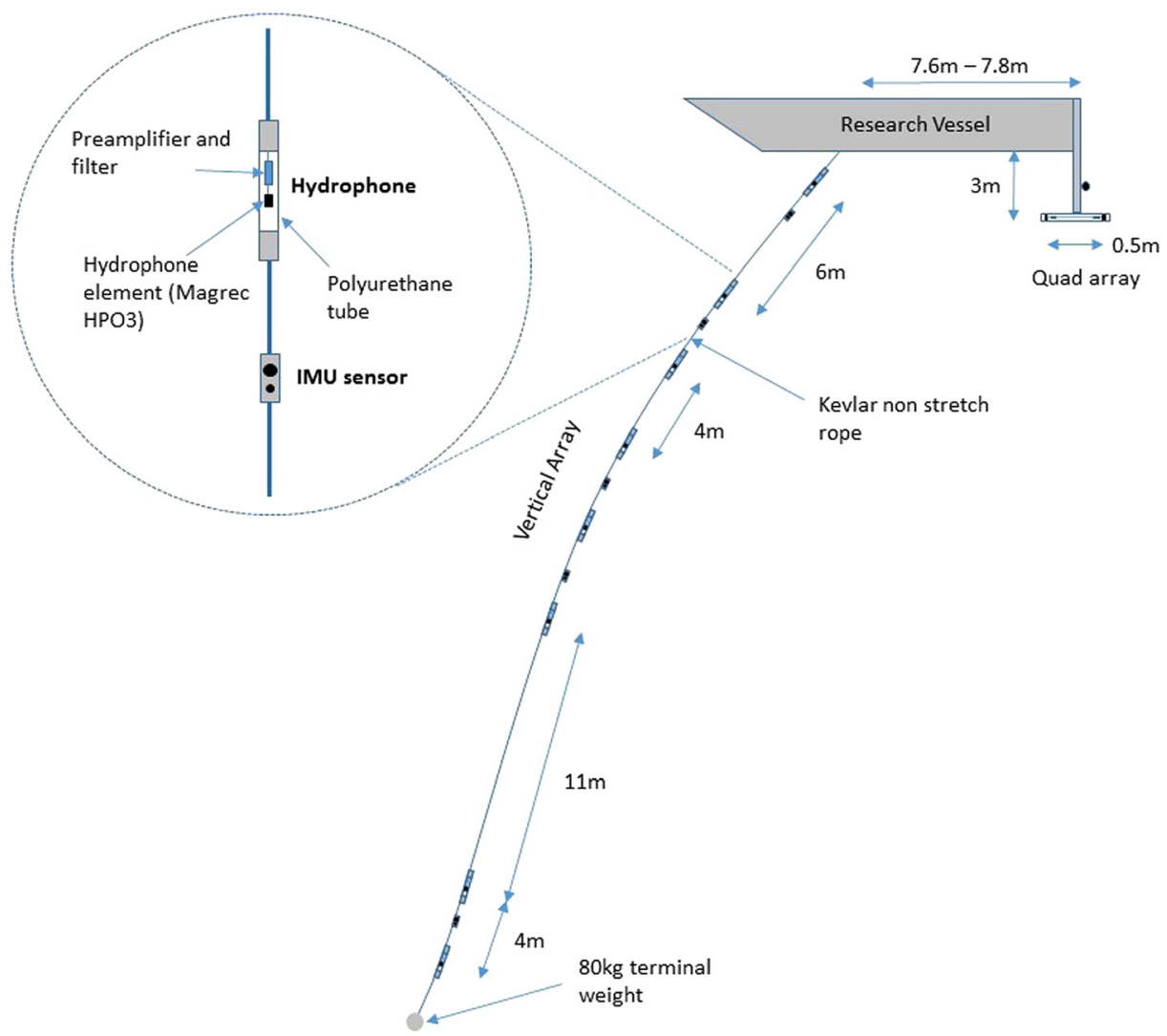

FIG. 2. (Color online) Diagram (not to scale) of the ten hydrophone large aperture array. The system consisted of both a flexible vertically orientated array with eight dispersed hydrophone elements and an additional rigid tetrahedral cluster (the quad array) which allowed the bearing to an animal to be calculated. When combined with information collected by a vector GPS and orientation sensors on the vertical array, this allowed geo-referenced positions of harbour porpoises to be determined. The array is shown at an angle to illustrate potential movement in a tidal stream. Unless otherwise indicated on the diagram, the distance between hydrophone elements on the vertical array was $4 \mathrm{~m}$.

$$
\hat{\mathbf{u}}_{i}=\frac{\mathbf{u}_{i}}{\left|\mathbf{u}_{i}\right|}
$$

where

$$
\mathbf{u}_{i}=\frac{\left(i-i_{A}\right)}{\left(i_{B}-i_{A}\right)}\left(\hat{\mathbf{u}}_{B}-\hat{\mathbf{u}}_{A}\right) .
$$

$\hat{\mathbf{u}}_{i}$ is the unit vector of section $i$ with $i=1,2,3, \ldots$, n. $i_{A}$ is the number of the first section above $i$ which holds a tag and $i_{B}$ is the number of the first section below $i$ which holds a tag. $\hat{\mathbf{u}}_{B}$ is the recorded unit vector of the tag on section $i_{B}$ and $\hat{\mathbf{u}}_{A}$ is the recorded unit vector of the tag on section $i_{A}$. Sections which have no tag above have a unit vector $\hat{\mathbf{u}}_{B}$ and sections which have no tag between them at the end of the array have a unit vector $\hat{\mathbf{u}}_{A}$.

Once the orientation angles for each section have been calculated, $(x y z)$ positions can be determined using the unit vector and length of each section

$$
\boldsymbol{v}_{i}=\hat{\mathbf{u}}_{i} \times C+\boldsymbol{v}_{i-1}
$$

where $v_{i}$ is the $(x, y, z)$ position of the end of each section and $C$ is the length of a section. This process is carried out for sections $0-n$ to determine the shape and orientation of the array. Data from the ship based inclinometer and vector GPS are then used to calculate the real world location (latitude, longitude, and height) of each $\left(x_{0}, y_{0}, z_{0}\right)$, and thus the real world location of all hydrophones on the array.

\section{Refraction}

Refraction of sound due to temperature and salinity gradients in underwater environments has the potential to introduce large errors into localisation. Strong turbulent water flows in tidal rapids usually result in well mixed water masses. Sound speed profiles were calculated for all survey locations using CTD profiles from the British Oceanographic Data Centre (BODC) database and potential refraction modelled using ACTUP Software (Duncan and Maggi, 2006). Refraction was insignificant and variation in speed of sound was no greater than $5.68 \mathrm{~ms}^{-1}$. This is negligible $(0.4 \%)$ and thus a conservative estimate of $10 \mathrm{~ms}^{-1}$ was added as an error to straight line sound speed calculations rather than using more complex refraction models for localisation (e.g., Thode, 2005).

\section{Noise}

To detect a harbour porpoise click, the received signal must be greater than the background noise level. Sediments moving in strong currents are thought to be a major source of noise in tidal areas (Bassett et al., 2013). There is no solution to high levels of noise if present in a frequency band of interest, however all hydrophones were calibrated, allowing for an assessment of the potential impacts on harbour porpoise detectability.

The maximum range at which an on-axis click with a source level of $191 \mathrm{~dB}$ re $\mu \mathrm{Pa}$ pp (the average source level of wild porpoise clicks; Villadsgaard et al., 2007) would be detected was calculated using a simple spherical propagation model:

$$
T L=20 \log _{10}(R)+\alpha R,
$$

where $T L$ is the transmission loss $R$ is the range in meters and $\alpha$ is the absorption coefficient. $\alpha$ was calculated to be 0.041 at 
$130 \mathrm{kHz}$ (Ainslie and Mccolm, 1998). The maximum detection range can be assumed to be the range $R$, at which

$$
S L-T L=N L+S N R
$$

where $S L$ is the source level (191 dB re $\mu \mathrm{Pa} \mathrm{pp}), N L$ is the noise level in the 100 to $160 \mathrm{kHz}$ frequency band and $S N R$ is the minimum signal to noise ratio at which clicks can be detected. This equation was solved for $R$ to assess the impact on porpoise detectability of variable noise conditions in tidal areas.

\section{Software}

\section{Click detection}

Raw acoustic data were passed through the PAMGuard click detector module with a signal to noise ratio (SNR) threshold set to $10 \mathrm{~dB}$. All detected clicks were classified as likely to be from a harbour porpoise or unidentified based on their spectral content and length using the PAMGuard click classifier (Gillespie et al., 2009).

\section{Localisation and tracking}

A time series of hydrophone positions, calculated in MATLAB using the methods detailed in Sec. III B 3, were then imported into PAMGuard. Detected porpoise clicks and hydrophone positions were used to calculate animal locations using a new PAMGuard localisation module which implemented the mimplex methods described above. The localisation modules assumed the standard error in sound speed was $10 \mathrm{~ms}^{-1}$, the standard error in hydrophone positions was $1 \mathrm{~cm}$ if on a flexible array, i.e., vertical array and $1 \mathrm{~mm}$ if rigid, i.e., between quad array hydrophones and the cross correlation error was assumed to be $2 \mu$ s (one sample).

Localisation results were filtered to remove any points above the sea surface and at ranges greater than $200 \mathrm{~m}$ as accuracy trials showed localised positions clearly broke down after this range. Finally, only results with a $\chi^{2}$ value of less than 250 were used in further analyses. This value was chosen as manual inspection of results showed it provided a good balance between excluding obviously incorrect localisations and removing too many correct values.

Harbour porpoise track fragments were then calculated in MATLAB from the filtered localisation results using the Kalman filter algorithm described in Sec. III B 2.

\section{Simulation of errors}

Error surfaces were generated to simulate the localisation accuracy of both the arrays at different ranges. The PAMGuard Sound Acquisition and Click Detector modules were used to generate simulated clicks in a $100 \mathrm{~m} \times 100 \mathrm{~m}$ grid around both arrays at five depths, 0, 10, 20, 30, and $40 \mathrm{~m}$. A simulated click on each of the hydrophones was generated assuming a source was located at every grid point and then localised using exactly the same methods applied to real data. The errors predicted by the mimplex algorithm were used to generate error surfaces.

\section{Noise}

Noise measurements were made using the PAMGuard Filtered Noise Measurement module. A bandpass $100 \mathrm{kHz}$ to $160 \mathrm{kHz}$, sixth order Butterworth filter (Butterworth, 1930) was used, with a measurement period of $10 \mathrm{~s}$. To provide a visual representation of noise, long term spectral averages (LTSA) were calculated using the PAMGuard LTSA modules, with bin size set to $60 \mathrm{~s}$.

\section{FIELD TRIALS}

Field work was carried out in coastal waters off the west of Scotland, in the Sound of Islay, the Great Race and Gulf of Corryvreckan in 2013 and in Kyle Rhea and the Sound of Sleat in 2014. The research vessel used was Silurian, a $16 \mathrm{~m}$ motor sailing vessel. Broadcast trials occurred in 2013.

\section{RESULTS}

\section{A. Localisation accuracy and mimplex performance}

During localisation accuracy trials, 39\% of detected broadcast clicks were successfully localised. If it is assumed that, with an omnidirectional sound source, half of the clicks detected are likely to be surface echoes, the proportion of direct path clicks localised accurately is likely to be between $70 \%$ and $80 \%$. Figures 3(a) and 3(b) show the localised depth and range of the sound source plotted against true depth and range. Figure 3(c) shows a summary of real and simulated errors; the median error in the field trials (difference between true and localised position) is plotted alongside simulated errors in $25 \mathrm{~m}$ range bins. The mean error in angle to the sound source was $3.06^{\circ}$. This was not affected by range.

\section{B. Noise}

Figure 4 shows an example of long term spectral averages over two $\sim 8 \mathrm{~h}$ long tidal surveys in the Sound of Islay and the Great Race. The line shows the expected on-axis detection range for a $191 \mathrm{~dB}$ re $1 \mu \mathrm{Pa}$ peak to peak porpoise click (the highest average recorded in the wild) assuming that detection is possible at a SNR of $10 \mathrm{~dB}$; this was the SNR used in the click detection algorithm. In the Sound of Islay, large broad band noise spikes are present, whilst the majority of ambient noise in the Great Race remains well below the porpoise frequency band.

\section{Click detection distribution}

A minimum of three true duplicated clicks must be detected on the vertical section of the array to allow the mimplex algorithm to calculate a location. For every successful localisation, the distance between the two most widely separated hydrophones on which the click was detected click was calculated. The results (Fig. 5) clearly show that, for most localisations, clicks were detected on widely separated hydrophones. 


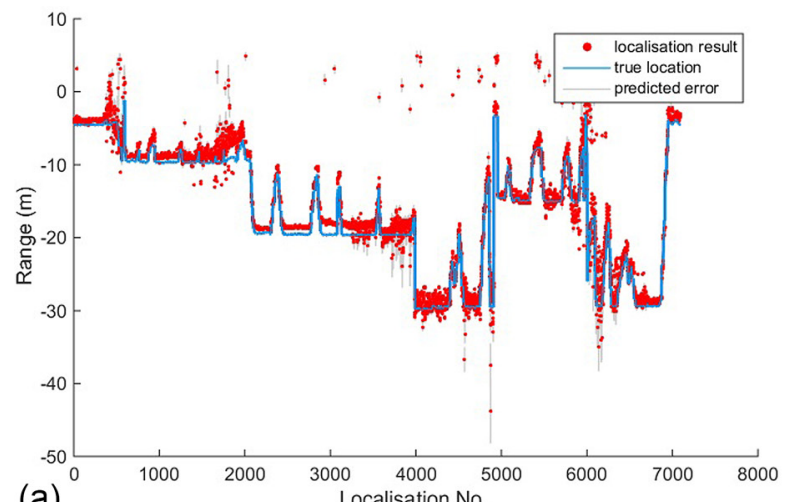

(a)
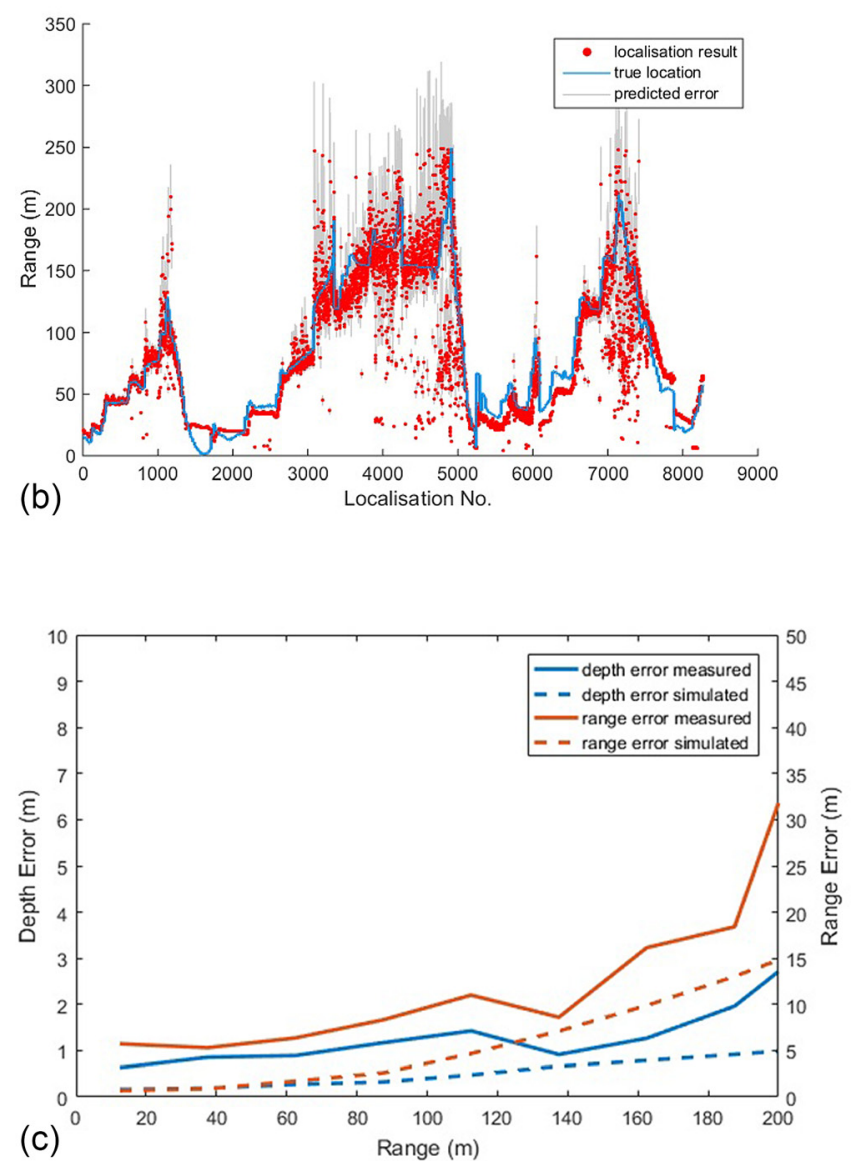

FIG. 3. (Color online) The localised depth and range of the sound source compared to the true location of the pinger and summary of errors. (a) and (b) The depth and range of the localised clicks compared to the true depth and range of the sound source. Error bars are 95\% confidence intervals calculated by the MCMC algorithm. (c) A plot of errors in depth and range against range and also shows predicted errors calculated from simulations. Errors in field measurements are the median difference between true location and calculated location and in simulation are the $95 \%$ confidence interval calculated by MCMC.

\section{Tracks}

Figure 6 shows an example of porpoise dive tracks from the Sound of Sleat, calculated using the mimplex and tracking algorithms. In total, $171 \mathrm{~h}$ of data was recorded and an average of 822170 porpoise clicks per hydrophone were detected. This resulted in 5206 geo-referenced track fragments.

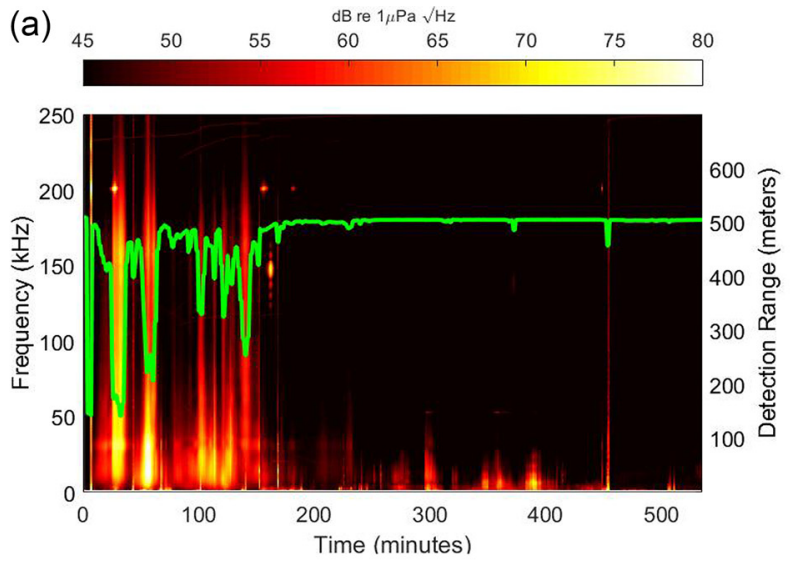

(b)

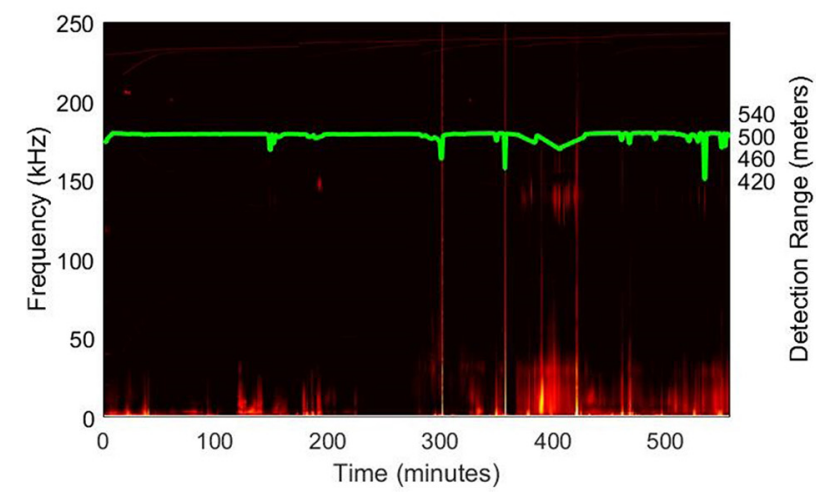

FIG. 4. (Color online) Long term spectral average and a measure of the corresponding detection range for a $191 \mathrm{~dB}$ re $1 \mu \mathrm{Pa}$ peak to peak on-axis porpoise click. Two full survey days in the Sound of Islay and the Great Race are shown as examples. The noise levels in the Sound of Islay were highly variable and broadband which resulted in large changes in the detection range during periods of noise, as shown with corresponding decreases in detection range. Noise was generally well below the porpoise frequency band in the Great Race, resulting in a much less variable detection range.

\section{DISCUSSION}

The large aperture hydrophone array developed here was designed specifically to obtain detailed behavioural information on harbour porpoises within tidal rapid sites and over the typical time scale of a survey ( $>1$ week). Results from field trials and surveys demonstrate that (1) large aperture arrays can coherently detect porpoise NBHF clicks on a

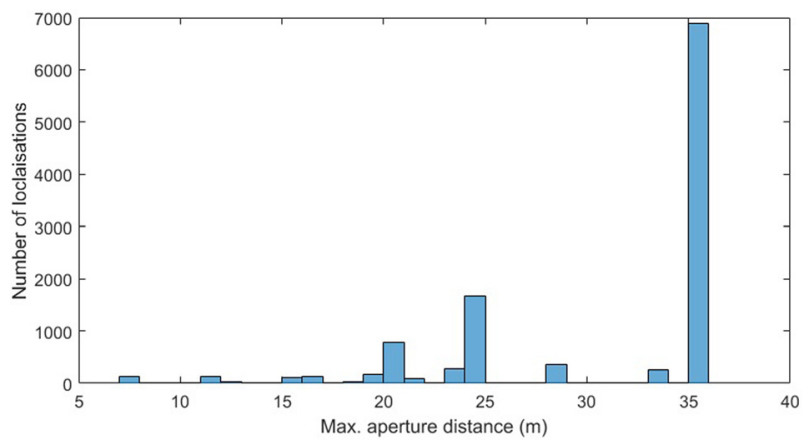

FIG. 5. (Color online) Histogram showing the distance between the two most widely separated hydrophones on the array (max aperture distance) on which a localised click was detected. The majority of porpoise detections which could be localised (i.e., detected on a minimum of three hydrophones) involve detections on widely spaced hydrophone elements. 

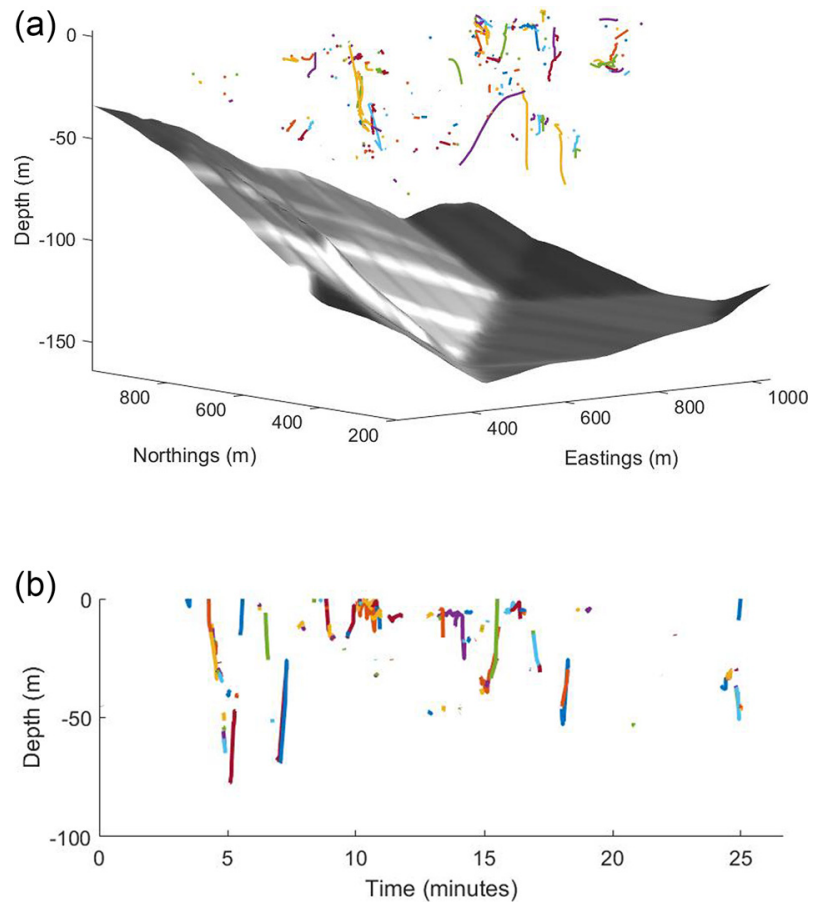

FIG. 6. (Color online) Example of dive fragments of harbour porpoises in the Sound of Sleat, Scotland. (a) shows an example of geo referenced tracks and bathymetry. (b) shows an example of track fragments in depth and time. Note size differences in fragments in both graphs. Tracks can be tens of seconds long, forming a significant portion of an animal's dive, or can be just a few seconds long, providing a "snapshot" location of an animal.

sufficient number of hydrophones to attempt localisation, (2) large aperture arrays are capable of providing accurate information on 3D animal locations in high current areas, and (3) effective automated methods can be used to analyse data.

The majority of published studies which have used PAM to determine the location of harbour porpoises have utilised small rigid hydrophone arrays with aperture sizes on the order of a few meters (e.g., Ural et al., 2006; Villadsgaard et al., 2007; Kyhn et al., 2013). Although appropriate for those studies, small hydrophone apertures significantly reduce the range at which accurate localisations are possible and therefore the efficacy of adopting such systems to measure dive profiles is limited. Results presented in this manuscript show that, despite the narrow beam profile and high attenuation of NBHF clicks, much larger hydrophone apertures can be used for localising wild harbour porpoises. The ability to do so greatly increases the potential range at which animals can be localised (here $\sim 200 \mathrm{~m}$ ) allowing these methods to be applied as an effective survey methodology to collect data on the fine scale behaviour of echolocating animals.

Many designs of vertical arrays were tested in tidal rapids while developing the array configuration described here. It became apparent during the development process that movement of the hydrophone array, due to the windage of the survey vessel and differential underwater currents, was introducing large localisation errors. Any movement of the vertical array results in uncertainties in hydrophone positions which then propagate to large errors in the localised positions of animals. To solve this, the shape of the array underwater was reconstructed using orientation units, allowing the positions of hydrophones to be accurately calculated. However, any vertical linear array which sits off the vertical angle also introduces fundamental ambiguities in localisation which cannot be resolved by knowledge of hydrophone positions. The addition of the quad array broke the linearity of the vertical array and so removed this fundamental ambiguity, recovering localisation accuracy and so allowing 3D positions of animals to be calculated. The localisation accuracy of the system was extensively tested both in the field during broadcast trials and using simulation tools. The field trials showed similar increases in error with range but, in general, average errors were around twice that of simulations. This could be caused by un-modelled errors in the array geometry calculations or the use of a constant or incorrect sound speed profile, which were not included in simulations but are almost certainly present when equipment is deployed in the field. Simulated data can therefore provide an initial estimate of the errors around an array but is not a substitute for testing in the field. Despite the increase in error, field trials demonstrated that, when combined with data from a vector GPS and orientation sensors, it was possible to obtain accurate geo-referenced localisations of the broadcast pinger, with sub-meter accuracy in depth at ranges $<60 \mathrm{~m}$. The vertical array is therefore capable of resolving fine scale behaviours of animals; However, to maintain such accuracy in high current areas is vital that both the movement of the array is accurately measured and, crucially, the array is designed in such a way that any linear symmetry is broken.

To make this a viable survey methodology, automated tools were required to analyse the large quantities of acoustic data collected over significant time periods (e.g., 14 days). Without running experiments with a tagged animal and accompanying PAM array, it is difficult to quantify the effectiveness of some aspects of the automated tracking system (hydrophone array + automated algorithms), e.g., how accurate the tracking algorithm is at separating multiple animals and what proportion of porpoise tracks are recorded at different ranges. However, it was possible to test the performance of the mimplex algorithm. Results from the click broadcast trials demonstrated the automated mimplex algorithm was likely able to accurately localise $\sim 70 \%$ to $80 \%$ of individual clicks. Considering that the average click rate of a wild harbour porpoises in the areas studies was $\sim 14$ clicks per second, this should allow measurement of fine scale movements of detected animals. The omni-directional source created multiple reverberations and echoes so the broadcast trials were a good test of the mimplex algorithm's ability to successfully match clicks on different hydrophones. Since the output source did not move like a porpoise, or simulate typical acoustic behaviour, the broadcast trials could not be used to test the tracking algorithm. Assessing tracking performance will be the focus of further research, however, visual inspection of tracking results indicated the algorithm produced realistic tracks from localised points. The ability of the mimplex algorithm to match clicks and the tracking algorithm to separate and interpolate individual dive fragments was particularly useful during the tidal rapid surveys. Patches of high frequency noise, shallow water leading to 
reverberation, and high densities of vocalizing porpoises produced a particularly complex soundscape in the porpoise frequency band and meant that simultaneous tracking of multiple individuals was often required. The automated nature of these methods is vital for surveys; manual attempts at matching and localising 822170 porpoise clicks would be both costly and prohibitively time consuming.

Although these methods provide an effective system for tracking animals, there are several limitations which must be considered. Noise is a major concern for any PAM survey and could conceivably reduce the average detection range of animals to an extent that makes PAM arrays ineffective in some areas. The tidal sites surveyed here had variable noise profiles. The most extreme example, the Sound of Islay [Fig. 4(a)], shows the maximum detection range of an on-axis click being reduced to $150 \mathrm{~m}$ in some parts of the tidal stream, a reduction of over $2 / 3$. This would reduce the number of detections by approximately $(2 / 3)^{2}$, a factor of 2.25 . Other studies have noted localised and often geographically consistent increases in high frequency noise levels in tidal rapids (Malinka et al., 2015; Gordon et al., 2011). The experience with these surveys is much the same, with occasional localised patches of high frequency noise in which porpoise detection probability will be substantially reduced. Therefore, a prudent approach before considering the use of complex drifting arrays or any other PAM system in a tidal rapid area would be to make measurements of ambient noise levels in the areas of interest, especially when tidal currents are strongest, to determine whether PAM surveys are a viable option.

The other significant consideration when employing this system, and indeed with PAM in general, is the strong association between the detectability of any animal with a narrow beam profile and its orientation, range and depth. An animal facing away from the array is far less likely to be detected than one facing towards the array. This problem is exacerbated when localisation, which requires detections on a number of hydrophones, is being attempted. Consequently, it is very rare for an entire dive to be recorded; instead fragments of animal tracks are produced. However, this is somewhat tempered by the ability of the system to collect large quantities of data on multiple animals which can be analysed to produce statistically meaningful measures of harbour porpoise behaviour and use of the water column.

A primary driver for this work was to better quantify the collision risk tidal turbines might pose to harbour porpoises. For this to be determined, substantial datasets on underwater behaviour must be collected from many animals, in multiple tidal areas and over full tidal and diel cycles. The drifting system and automated algorithms described in this paper can be targeted at specific geographic areas such as tidal rapids, provide accurate information on animal behaviour and is cost effective, so is well suited to this task. Although developed primarily for tracking harbour porpoises in tidal rapids, the methods developed here are relatively general and could be applied to other echolocating species, other habitats and/ or array types. Indeed, the relatively high attenuation and directionality of NBHF clicks makes the harbour porpoise a particularly poor candidate species for localisation; it is therefore to be expected that this methodology may work more effectively with other echolocating species in less energetic habitats.

\section{CONCLUSION}

Information on the fine-scale movements of animals underwater has primarily been the preserve of tagging, and indeed the information that tagged animals have provided has been instrumental in our understanding of animal behaviour. However, in the specific case of a small geographic area of interest, such as tidal streams, the cost/data ratio of tagging animals, which may spend the majority of their time outside these areas, is less favourable. We have shown that, in these situations, localisation using large aperture drifting arrays is a viable alternative methodology and can be used effectively in tidal rapid habitats. It provides a targeted, costeffective and non-invasive platform to provide high resolution data on animal behaviour and can be utilised in adverse conditions during both day and night.

\section{ACKNOWLEDGMENTS}

Thanks to Carol Sparling and Gordon Hastie of SMRU, and Ben Wilson and Steven Benjamins of SAMS for input and helpful discussions. Special thanks to Alex Coram (SMRU) for practical input, field assistance and discussion throughout this project, as well as other field assistants over the years. We are grateful to Jay Barlow for suggesting the use of the Sony XPLOD for this application. Thanks to "Student Dave Tutorials" for code resources and instructive tutorials and BODC for supplying CTD profiles. This work was funded by the Scottish Government through the Marine Mammal Scientific Support Research Programme MMSS/001/11.

\footnotetext{
${ }^{1}$ See supplementary material at http://dx.doi.org/10.1121/1.4976077 for an animation showing the movement of the hydrophone array in a tidal stream and the MATLAB code to calculate harbour porpoise tracks from localisation points.
}

Ainslie, M. A., and Mccolm, J. G. (1998). "A simplified formula for viscous and chemical absorption in sea water," J. Acoust. Soc. Am. 103(3), $1671-1672$.

Au, W. W. L., and Hastings, M. C. (2008). "Signal recording and data acquisition," in Principles of Marine Bioacoustics (Springer, New York), Chap. 5, pp. 155-168.

Au, W. W. L., Kastelein, R. A., Rippe, T., and Schooneman, N. M. (1999). "Transmission beam pattern and echolocation signals of a harbor porpoise (Phocoena phocoena)," J. Acoust. Soc. Am. 106(6), 3699-3705.

Bassett, C., Thomson, J., and Polagye, B. (2013). "Sediment-generated noise and bed stress in a tidal channel," J. Geophys. Res. Oceans 118(4), 2249-2265.

Benjamins, S., Dale, A., Hastie, G., Waggitt, J., Lea, M.-A., Scott, B., and Wilson, B. (2015). "Confusion reigns? A review of marine megafauna interactions with tidal-stream environments," Oceanogr. Mar. Biol. 53, 1-54.

Berclaz, J., Fleuret, F., Türetken, E., and Fua, P. (2011). "Multiple object tracking using k-shortest paths optimization," IEEE Trans. Pattern Analysis Machine Intell. 33(9), 1806-1819.

Butterworth, S. (1930). "On the theory of filter amplifiers," Exp. Wireless Wireless Eng. 7, 536-541.

Chib, S., and Greenberg, E. (1995). "Understanding the Metropolis-Hastings algorithm," Am. Stat. 49(4), 327-335.

Deruiter, S. L., Bahr, A., Blanchet, M.-A., Hansen, S. F., Kristensen, J. H., Madsen, P. T., Tyack, P. L., and Wahlberg, M. (2009). "Acoustic behaviour of echolocating porpoises during prey capture,” J. Exp. Biol. 212(19), 3100-3107. 
Duncan, A. J., and Maggi, A. L. (2006). "A consistent, user friendly interface for running a variety of underwater acoustic propagation codes," in Proceedings of the First Australasian Acoustical Societies' Conference, Christchurch, New Zealand, pp. 471-477.

European Commission (1992). "Council Directive 92/43/EEC of 21 May 1992 on the conservation of natural habitats and of wild fauna and flora," OJ L 206, pp. 7-50, available at http://eur-lex.europa.eu/legal-content/EN/ TXT/?uri=CELEX:31992L0043 (Last viewed January 2017).

Frid, C., Andonegi, E., Depestele, J., Judd, A., Rihan, D., Rogers, S. I., and Kenchington, E. (2012). "The environmental interactions of tidal and wave energy generation devices," Environ. Impact Assess. Rev. 32(1), 133-139.

Gillespie, D., Gordon, J., Mchugh, R., Mclaren, D., Mellinger, D., Redmond, P., Thode, A., Trinder, P., and Deng, X. Y. (2009). "PAMGUARD: Semiautomated, open source software for real-time acoustic detection and localisation of cetaceans," J. Acoust. Soc. Am. 125, 2547.

Goodwin, L. (2008). "Diurnal and tidal variations in habitat use of the harbour porpoise (Phocoena phocoena) in Southwest Britain," Aquat. Mamm. 34(1), 44-53.

Gordon, J., Thompson, D., Leaper, R., Gillespie, D., Pierpoint, C., Calderan, S., Macaulay, J., Gordon, T., and Simpson, N. (2011). "Assessment of risk to marine mammals from underwater marine renewable devices in Welsh waters - Phase 2 - Studies of marine mammals in Welsh high tidal waters," Welsh Assembly Government, Cardiff, Wales.

Hammond, P. S., Macleod, K., Berggren, P., Borchers, D. L., Burt, L., Cañadas, A., Desportes, G., Donovan, G. P., Gilles, A., Gillespie, D., Gordon, J., Hiby, L., Kuklik, I., Leaper, R., Lehnert, K., Leopold, M., Lovell, P., Oien, N., Paxton, C. G. M., Ridoux, V., Rogan, E., Samarra, F., Scheidat, M., Sequeira, M., Siebert, U., Skov, H., Swift, R., Tasker, M. L., and Teilmann, J. (2013). "Cetacean abundance and distribution in European Atlantic shelf waters to inform conservation and management," Biol. Conser. 164, 107-122.

Hastie, G. D., Wilson, B., and Thompson, P. M. (2006). "Diving deep in a foraging hotspot: Acoustic insights into bottlenose dolphin dive depths and feeding behaviour," Mar. Biol. 148(5), 1181-1188.

Heerfordt, A., Møhl, B., and Wahlberg, M. (2007). "A wideband connection to sperm whales: A fiber-optic, deep-sea hydrophone array," Deep Sea Res. Part I 54(3), 428-436.

Holt, M. M., Noren, D. P., Veirs, V., Emmons, C. K., and Veirs, S. (2009). "Speaking up: Killer whales (Orcinus orca) increase their call amplitude in response to vessel noise," J. Acoust. Soc. Am. 125, EL27-L32.

Kalman, R. E. (1960). "A new approach to linear filtering and prediction problems," Trans. ASME J. Basic Eng. 82, 35-45.

Koblitz, J. C., Wahlberg, M., Stilz, P., Madsen, P. T., Beedholm, K., and Schnitzler, H.-U. (2012). "Asymmetry and dynamics of a narrow sonar beam in an echolocating harbor porpoise," J. Acoust. Soc. Am. 131(3), 2315-2324.

Kuhn, H. W. (1955). "The Hungarian method for the assignment problem," Naval Res. Logistics Quart. 2(1-2), 83-97.

Kyhn, L. A., Tougaard, J., Beedholm, K., Jensen, F. H., Ashe, E., Williams, R., and Madsen, P. T. (2013). "Clicking in a killer whale habitat: Narrowband, high-frequency biosonar clicks of harbour porpoise (Phocoena phocoena) and Dall's Porpoise (Phocoenoides dalli)," PLoS ONE 8(5), e63763.

Linnenschmidt, M., Teilmann, J., Akamatsu, T., Dietz, R., and Miller, L. A. (2013). "Biosonar, dive, and foraging activity of satellite tracked harbor porpoises (Phocoena phocoena),” Mar. Mamm. Sci. 29(2), E77-E97.

Luetteke, F., Zhang, X., and Franke, J. (2012). "Implementation of the Hungarian Method for object tracking on a camera monitored transportation system," in 7th German Conference on Robotics, pp. 1-6.

MacQueen, J. B. (1967). "Kmeans some methods for classification and analysis of multivariate observations," in 5th Berkeley Symposium on Mathematical Statistics and Probability, Vol. 1, No. 233, pp. 281-297.

Malinka, C. E., Hay, A. E., and Cheel, R. (2015). "Towards acoustic monitoring of marine mammals at a tidal energy site: Grand Passage, NS, Canada," in Proceedings of the 11th European Wave and Tidal Energy Conference, pp. 1-10.

Marubini, F., Gimona, A., Evans, P. G. H., Wright, P. J., and Pierce, G. J. (2009). "Habitat preferences and interannual variability in occurrence of the harbour porpoise phocoena phocoena off northwest Scotland," Mar. Ecol. Prog. Ser. 381, 297-310.

Metropolis, N., Rosenbluth, A. W., Rosenbluth, M. N., Teller, A. H., and Teller, E. (1953). "Equation of state calculations by fast computing machines," J. Chem. Phys. 21(6), 1087-1092.
Miller, B., and Dawson, S. (2009). "A large-aperture low-cost hydrophone array for tracking whales from small boats," J. Acoust. Soc. Am. 126(5), 2248-2256.

MMPA (1972). Marine Mammal Protection Act of 1972. 16 U.S.C. et seq. and 50 CFR Part 216.

Mohl, B., and Andersen, S. (1973). "Echolocation: high-frequency component in the click of the Harbour Porpoise (Phocoena ph. L.)," J. Acoust. Soc. Am. 54(5), 1368-1372.

Møhl, B., Wahlberg, M., Madsen, P. T., Miller, L. A., and Surlykke, A. (2000). "Sperm whale clicks: Directionality and source level revisited," J. Acoust. Soc. Am. 107(1), 638-648.

Nelder, J. A., and Mead, R. (1965). "A simplex method for function minimization," Comput. J. 7(4), 308-313.

Pierpoint, C. (2008). "Harbour porpoise (Phocoena phocoena) foraging strategy at a high energy, near-shore site in south-west Wales, UK," J. Mar. Biol. Assoc. U. K. 88(6), 1167-1173.

Press, W. H., Teukolsky, S. A., Vetterling, W. T., and Flannery, B. P. (1988). "Minimization or maximization of functions," in Numerical Recipes: The Art of Scientific Computing, 2nd ed. (Cambridge University Press, New York), Chap. 10, pp. 408-412.

Teilmann, J., Tougaard, J., Miller, L. A., Kirketerp, T., Hansen, K., and Brando, S. (2006). "Reactions of captive harbor porpoises (Phocoena phocoena) to pinger-like sounds," Mar. Mamm. Sci. 22(2), 240-260.

Thode, A. (2005). "Three-dimensional passive acoustic tracking of sperm whales (Physeter macrocephalus) in ray-refracting environments," J. Acoust. Soc. Am. 118(6), 3575-3584.

Thompson, D., and Lonergan, M. (2015). "Collision risk and impact Study: Examination of models for estimating the risk of collisions between seals and tidal turbines," Report MR 7.2.2, Sea Mammal Research Unit, University of St Andrews, St Andrews, UK.

Toke, D. (2011). "The UK offshore wind power programme: A sea-change in UK energy policy?," Energy Policy 39(2), 526-534.

Ural, T., Bahli, R., Yano, M., Inoue, T., Sakamaki, T., and Fukuchi, T. (2006). "Results from a high-resolution acoustic device for monitoring finless porpoises in coastal precincts off Japan," in OCEANS 2006 - Asia Pacific, pp. 1-5.

Verfuß, U. K., Miller, L. A., Pilz, P. K. D., and Schnitzler, H.-U. (2009). "Echolocation by two foraging harbour porpoises (Phocoena phocoena)," J. Exp. Biol. 212, 823-834.

Villadsgaard, A., Wahlberg, M., and Tougaard, J. (2007). "Echolocation signals of wild harbour porpoises, Phocoena phocoena," J. Exp. Biol. 210(1), $56-64$.

Wahlberg, M., Møhl, B., and Madsen, P. T. (2001). "Estimating source position accuracy of a large-aperture hydrophone array for bioacoustics," J. Acoust. Soc. Am. 109(1), 397-406.

Waring, G. T., Josephson, E., and Maze-foley, K. (2015). "US Atlantic and Gulf of Mexico Marine Mammal Stock Assessments - 2014," NOAA Tech Memo NMFS NE 231, 36 p.

Watkins, W. a., and Schevill, W. E. (1972). "Sound source location by arrival-times on a non-rigid three-dimensional hydrophone array," Deep Sea Res. Oceanogr. Abstr. 19(10), 691-706.

Wiggins, S. M., McDonald, M. A., and Hildebrand, J. A. (2012). "Beaked whale and dolphin tracking using a multichannel autonomous acoustic recorder," J. Acoust. Soc. Am. 131(1), 156-163.

Wilson, B., Batty, R. S., Daunt, F., and Carter, C. (2007). "Collision risks between marine renewable energy devices and mammals, fish and diving birds," Report PA37 1QA, Scottish Association for Marine Science, Oban, Scotland.

Wisniewska, D. M. M., Johnson, M., Teilmann, J., Rojano-Doñate, L., Shearer, J., Sveegaard, S., Miller, L. A. A., Siebert, U., and Madsen, P. T. T. (2016). "Ultra-high foraging rates of harbor porpoises make them vulnerable to anthropogenic disturbance," Curr. Biol. 26(11), $1441-1446$.

Wisniewska, D. M., Ratcliffe, J. M., Beedholm, K., Christensen, C. B., Johnson, M., Koblitz, J. C., Wahlberg, M., and Madsen, P. T. (2015). "Range-dependent flexibility in the acoustic field of view of echolocating porpoises (Phocoena phocoena)," eLife 4, e05651.

Yussiff, A.-L., Yong, S.-P., and Baharudin, B. B. (2014). "Parallel Kalman filter-based multi-human tracking in surveillance video," in 2014 International Conference on Computer and Information Sciences (ICCOINS), pp. 1-6.

Zimmer, W. (2011). Passive Acoustic Monitoring of Cetaceans (Cambridge University Press, Cambridge, UK), 356 pp. 